\title{
Processing and application of nuclear data for low temperature criticality assessment
}

\author{
Tim Ware ${ }^{1, *}$, David Hanlon ${ }^{1}$, Tara Hanlon $^{1}$, Richard Hiles $^{1}$, Malcolm Lingard ${ }^{1}$, Ray Perry ${ }^{1}$, and Simon Richards ${ }^{1}$ \\ ${ }^{1}$ Wood, Kings Points House, Queen Mother Square, Poundbury, Dorchester, Dorset, DT1 3BW, UK
}

\begin{abstract}
Until recently, criticality safety assessment codes had a minimum temperature at which calculations can be performed. Where criticality assessment has been required for lower temperatures, indirect methods, including reasoned argument or extrapolation, have been required to assess reactivity changes associated with these temperatures.

The ANSWERS Software Service MONK ${ }^{\circledR}$ version 10B Monte Carlo criticality code, is capable of performing criticality calculations at any temperature, within the temperature limits of the underlying nuclear data in the BINGO continuous energy library. The temperature range of the nuclear data has been extended below the traditional lower limit of $293.6 \mathrm{~K}$ to $193 \mathrm{~K}$ in a prototype BINGO library, primarily based on JEFF-3.1.2 data. The temperature range of the thermal bound scattering data of the key moderator materials was extended by reprocessing the NJOY LEAPR inputs used to produce bound data for JEFF-3.1.2 and ENDF/B-VIII.0.

To give confidence in the low temperature nuclear data, a series of MONK and MCBEND calculations have been performed and results compared against external data sources. MCBEND is a Monte Carlo code for shielding and dosimetry and shares commonalities to its sister code MONK including the BINGO nuclear data library. Good agreement has been achieved between calculated and experimental cross sections for ice, k-effective results for low temperature criticality benchmarks and calculated and experimentally determined eigenvalues for thermal neutron diffusion in ice.

To quantify the differences between ice and water bound scattering data a number of MONK criticality calculations were performed for nuclear fuel transport flask configurations. The results obtained demonstrate good agreement with extrapolation methods. There is a discernible difference in the use of ice and water data.
\end{abstract}

\section{Introduction}

Until recently, criticality safety assessment codes had a minimum temperature at which calculations could be performed. Assessments for lower temperatures used indirect methods, such as reasoned argument or extrapolation, to assess reactivity changes associated with these temperatures.

However, driven by the International Atomic Energy Agency's transport regulation requirements to demonstrate criticality safety of a nuclear material transport package down to $-40^{\circ} \mathrm{C}$, direct methods of criticality assessment have been developed.

The ANSWERS Monte Carlo code MONK [1] uses the BINGO continuous energy nuclear data libarary and associated collision processor. Since MONK version 10B, the temperature of calculations is only limited by the temperatures tabulated on the BINGO library. With BINGO libraries, MONK includes a temperature interpolation capability for free gas nuclides based on runtime Doppler broadening. MONK version 11 A (in development) also includes stochastic interpolation of the $\mathrm{S}(\alpha, \beta)$ data thereby providing full flexibility in the temperature dependence.

*e-mail: tim.ware@woodplc.com

\section{Prototype BINGO Library Production}

The temperature range of the BINGO nuclear data library was extended via modification of the existing BINGO library generation route, which is described in further detail in [2]. Evaluated nuclear data are processed using the BINGO pre-processor (BPP). The BPP calls NJOY2016 [3] to generate point cross sections using the RECONR and BROADR modules. The remaining processing is performed internally by the BPP. The secondary angle and energy data for emitted neutrons, including the $\mathrm{S}(\alpha, \beta)$ thermal scattering data, are transformed to ease sampling, allowing the Monte Carlo code to generate secondary neutron emission data during the run.

Extending the temperature range of the free gas cross section data is straightforward; NJOY is requested to generate cross sections for the addtional temperatures. The $0 \mathrm{~K}$ cross sections reconstructed by RECONR are Doppler broadened to the extended temperature set in BROADR. The JEFF-3.1.2 evaluation was used to generate the majority of the free gas data. The new lower temperature limit of the BINGO free gas data was $193 \mathrm{~K}$ (plus $115 \mathrm{~K}$ for ${ }^{235} \mathrm{U}$ - see Section 3.1).

The extension of the temperature range of the bound thermal scattering data is more complex. The existing generation route was limited to the temperatures for which the 
thermal scattering data are provided in the evaluated files. Traditionally, the lowest evaluated temperature has been $293.6^{\circ} \mathrm{C}$. This temperature range was extended by regenerating the ENDF files using the NJOY LEAPR module. LEAPR inputs were obtained from;

- IKE: ${ }^{1} \mathrm{H}$ in $\mathrm{H}_{2} \mathrm{O}$ and ${ }^{1} \mathrm{H}$ in $\mathrm{ZrH}$ [4], $\mathrm{C}$ in graphite [5]

- ENDF/B-VIII.0: ${ }^{1} \mathrm{H}$ in $\mathrm{CH}_{2},{ }^{1} \mathrm{H}$ and ${ }^{16} \mathrm{O}$ in ice(Ih) [6]

The data published by Institut für Kernenergetik und Energiesysteme (IKE) is that used to produce the corresponding JEFF-3.1.2 thermal scattering evaluated files. One new temperature of $273.15 \mathrm{~K}$ was added for ${ }^{1} \mathrm{H}$ in $\mathrm{H}_{2} \mathrm{O}$. New temperatures down to $193 \mathrm{~K}$ were added for ${ }^{1} \mathrm{H}$ in $\mathrm{ZrH}$ and $\mathrm{C}$ in graphite. The three new ENDF/BVIII.0 LEAPR inputs already have data down to $115 \mathrm{~K}$; these inputs were rerun to customise the temperatures in the ENDF files.

There exist other bound nuclide data on the BINGO library. However, for this prototype library, the temperature ranges for these data were not extended. This will be done in future "production" BINGO libraries following similar methods to those described here.

For ${ }^{1} \mathrm{H}$ in $\mathrm{H}_{2} \mathrm{O}$, the continuous frequency spectrum is temperature dependent. In fact, the spectra published in [4] are interpolated from experimental data at $294 \mathrm{~K}$ and $624 \mathrm{~K}$ from [7]. A continuous frequency spectrum for $273.15 \mathrm{~K}$ was extrapolated from a fit to the published spectra. Other temperature dependent parameters (such as the translational weight) were then recalculated for $273.15 \mathrm{~K}$ from the normalisation of the continous spectrum.

For the remaining bound nuclides, the continuous frequency spectrum is not temperature dependent; the new required temperatures were simply input to LEAPR.

\section{Validation}

A key aspect of criticality safety assessment is the validation of the methods, codes and data underpinning the assessment. For validation against criticality experiments, benchmark data currently exist for $293.6 \mathrm{~K}$ and higher temperatures. In order to give confidence in the nuclear data below room temperature, particularly the recently evaluated ${ }^{1} \mathrm{H}$ in ice(Ih) bound scattering data, three comparisons were performed between the extended BINGO data library and independent data sets.

\subsection{Total Ice Cross Section}

Experimentally determined total cross sections for ice at $115 \mathrm{~K}$ were reported in [8]. Cross section values and statistical uncertainties were retrieved from EXFOR [9] for 117 neutron energy points. The experimental statistical uncertainties are up to $0.8 \%$ of the cross section value. The quoted uncertainty on the ice sample thickness puts a $0.4 \%$ systematic uncertainty on the cross section. Furthermore, the experimental data are normalised to the ${ }^{1} \mathrm{H}$ free gas scattering cross section from ENDF/B-VII.1, which has an uncertainty of over $2 \%$. Therefore, the total experimental uncertainty is $2.4 \%-2.6 \%$.
Additionally, calculated total cross sections for ice were reported in [10]. These are directly derived from the new $\mathrm{S}(\alpha, \beta)$ parameters published in ENDF/B-VIII.0. These calculated values have been estimated from the plotted data in [10].

A MONK calculation was performed for a pure ${ }^{235} \mathrm{U}$ pincell surrounded by ice. The "XT" feature of MONK provides the capability to obtain cross sections in userspecified energy groups based on scored reaction rates. The scored partial cross sections and scattering matrices for ${ }^{1} \mathrm{H}$ in ice(Ih) and ${ }^{16} \mathrm{O}$ in ice(Ih) were summed to produce a total cross section for ice.

Figure 1 compares the experimental cross section (crosses with errors), the calculated cross section of [10] (circles) and the MONK cross section (solid line). The MONK (C-E)/E is given on the secondary axis.

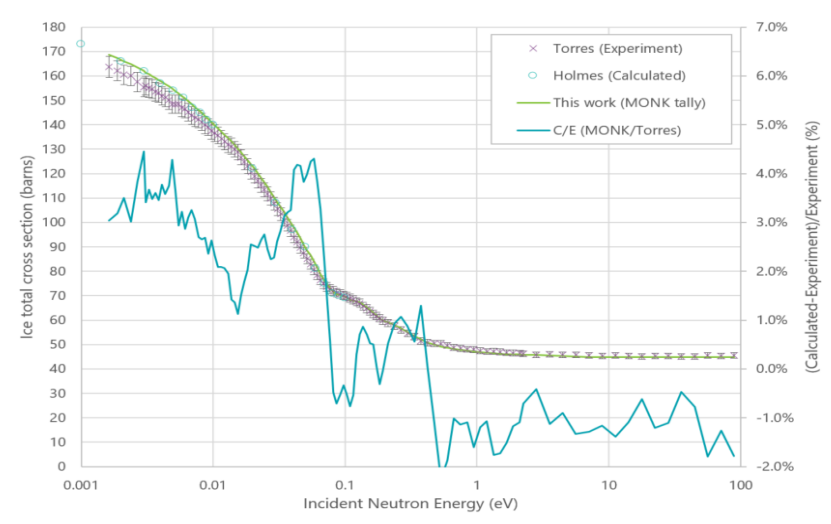

Figure 1. Comparison of ice cross sections.

There is excellent agreement between the two calculated cross sections. This demonstrates that two independent data processing routes produce practically equivalent cross sections. There is reasonable agreement between the calculations and the experimental cross section. It is proposed in [10] that the differences, which are within the experimental uncertainty, are due to the use of the incoherent approximation in calculating the elastic scattering in oxygen. This leads to overestimate of the cross section at lower energies but agreement with data in [11] make the incoherent approximation acceptable.

\subsection{Benchmark Comparison}

Calculations of the NEA BUC/Phase VII benchmark [12] using the MCNP code were reported in [13]. This computational benchmark uses a realistic model of a nuclear fuel flask composed of an array of PWR fuel rod assemblies immersed in water within a stainless steel cask. Comparing independent codes provides confidence in the results. The fuel is "at discharge" so contains 11 actinides. Ref. [13] gives results for uniform temperatures from 233 to $323 \mathrm{~K}$. Water is used for $277 \mathrm{~K}$ and above. For $273 \mathrm{~K}$ and below, calculations are performed with ice thermal scattering and temperature-dependent ice density, and also with ice thermal scattering data and fixed water density.

Figure 2 shows the MONK results using the extended BINGO library (stochastic uncertainty $=20 \mathrm{pcm}(1 \sigma)$ ). 
The three trend lines show different models for water; water thermal scattering data at water density at temperatures of $273.15 \mathrm{~K}$ and above, ice data with water density (as at $0^{\circ} \mathrm{C}$ ), at temperatures of $273.15 \mathrm{~K}$ and below, and ice data at ice density at temperatures of $273.15 \mathrm{~K}$ and below. At $273.15 \mathrm{~K}$, the difference between the water and ice data alone (i.e. same density) is $100 \pm 30 \mathrm{pcm}$. However, the change in density between ice and water has a much larger effect.

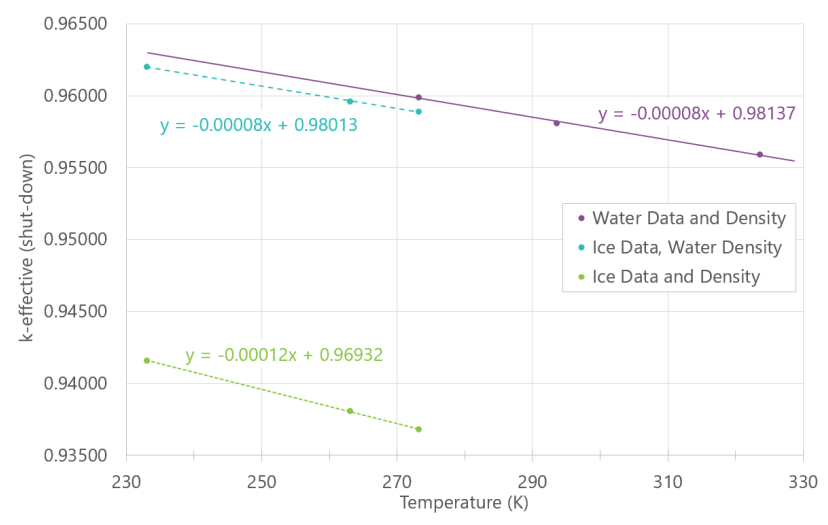

Figure 2. k-effectives for temperature dependent water data.

Despite using different data libraries ([13] used the pre-release JEFF-3.3T3 library), there is good agreement in the trends of these results and those plotted on page 10 of [13]. In both figures, the gradient of ice data \& density line is steeper than of the ice data $\&$ water density line. This is due to the ice density being temperature dependent which is significant.

\subsection{Decay of Neutron Pulse}

A series of neutron diffusion experiments in ice were reported in [11] and [14]. The measurement of the decaying thermal neutron flux following a mono-energetic pulsed neutron beam allows for the determination of the neutron diffusion parameters. The experiment was performed for cylinders of pure ice with a range of heights and radii, at temperatures from $-85^{\circ} \mathrm{C}$ to $0^{\circ} \mathrm{C}$.

Following a pulse of neutrons from the source, the high energy flux decays rapidly and after sufficient time, the flux will be in thermal equilibrium. The decay time of the neutron flux can then be approximated by the one-speed diffusion model as follows;

$$
\phi(r, t)=\phi_{0}(r) \exp (-\alpha t)
$$

where: $\phi$ is the flux, $r$ is the spatial position, $t$ is the time elapsed since flux $=\phi_{0}$ and;

$$
\alpha=v \Sigma_{a}+v D B^{2}-C B^{4}
$$

where: $\Sigma_{a}$ is the macroscopic absorption cross section, $v$ is the effective average neutron velocity, $D$ is the diffusion coefficient (i.e. $1 / \Sigma_{\text {transport }}$ ), $B^{2}$ is the geometric buckling, and $C$ is the diffusion cooling coefficient.
The diffusion is sensitive to both the total and differential thermal scattering cross sections. The absorption cross section is the dominant term for the larger cylinders where $B^{2}$ is small. The thermal scattering is dominant for small cylinders with larger $B^{2}$. The cylinder dimensions and $B^{2}$ values are taken from [11] and given in Table 1.

In the experiment the values of $\alpha$ are determined from the measured flux. For the comparison, the ANSWER's Monte Carlo code for shielding and dosimetry, MCBEND [1], was used to model the experiment and score the neutron flux following the neutron pulse. MCBEND includes a time dependence feature that allows the duration of the neutron pulse and the subsequent time bins for the scoring of the neutron flux to be easily specified. MCBEND has many commonalities with MONK including the processing of the BINGO nuclear data library and particle tracking.

As the temperatures of the data on the BINGO library are slightly different from the experiment temperatures, pairs of MCBEND calculations were performed at adjacent library temperatures but using the same ice material density corresponding to the experiment temperature. The $\alpha$ eigenvalues were determined from fits to the scored fluxes and these were interpolated to give the $\alpha$ 's corresponding to the experiment temperatures.

For each cylinder, Table 2 gives the MCBENDderived $\alpha$ values and the $\mathrm{C} / \mathrm{E}$ comparison against the experimentally-derived values. These results are in good agreement with the experimental data and the differences are within $3 \sigma$ (with one exception). In addition, the MCBEND results are in agreement with equivalent results reported in [10] giving confidence that the original ice $\mathrm{S}(\alpha, \beta)$ data has been processed and used by two independent code packages to give statistically equivalent results.

\section{Application Calculations}

MONK criticality calculations were performed to estimate the effect of the low temperature nuclear data, in particular that of ice, for nuclear fuel transport flask configurations. These trends show that ice should be explicitly modelled. However, they are indicative and must not be assumed to apply universally to any configuration.

\subsection{AGR Flooded Fuel Flask}

An accurate model of AGR-type reactor fuel elements in a fully-flooded fuel transport flask with thermal expansion applied to all materials showed that the reduction in $\mathrm{k}$ effective with increasing temperature is principally due the change in density of water and the ${ }^{238} \mathrm{U}$ neutron capture. Decreasing the temperature below $273.15 \mathrm{~K}$ decreases keffective primarily due to the $8 \%$ reduction in water density but also a small reduction due to changing the ice thermal scattering data. In terms of criticality assessment, the directly calculated k-effective using the ice data is close, to but lower than, results extrapolated from calculations at and above $293.6 \mathrm{~K}$ using water data. 
Table 1. Ice cylinder sizes and buckling values.

\begin{tabular}{c|cc|cc|cc|cc|cc|cc}
\hline & height & radius & \multicolumn{2}{|c|}{$-5^{\circ} \mathrm{C}$} & \multicolumn{2}{|c|}{$-25^{\circ} \mathrm{C}$} & \multicolumn{2}{|c|}{$-45^{\circ} \mathrm{C}$} & \multicolumn{3}{|c|}{$-65^{\circ} \mathrm{C}$} & \multicolumn{2}{c}{$-85^{\circ} \mathrm{C}$} \\
Cyl. & $(\mathrm{cm})$ & $(\mathrm{cm})$ & $\mathrm{B}^{2}\left(\mathrm{~cm}^{-2}\right)$ & $\alpha\left(\mathrm{s}^{-1}\right)$ & $\mathrm{B}^{2}\left(\mathrm{~cm}^{-2}\right)$ & $\alpha\left(\mathrm{s}^{-1}\right)$ & $\mathrm{B}^{2}\left(\mathrm{~cm}^{-2}\right)$ & $\alpha\left(\mathrm{s}^{-1}\right)$ & $\mathrm{B}^{2}\left(\mathrm{~cm}^{-2}\right)$ & $\alpha\left(\mathrm{s}^{-1}\right)$ & $\mathrm{B}^{2}\left(\mathrm{~cm}^{-2}\right)$ & $\alpha\left(\mathrm{s}^{-1}\right)$ \\
\hline 1 & 24.95 & 15.13 & 0.0393 & 5886 & 0.0393 & 5724 & 0.0394 & 5630 & 0.0395 & 5579 & 0.0396 & 54433 \\
2 & 18.54 & 12.55 & 0.0618 & 6478 & 0.0618 & 6387 & 0.0621 & 6207 & 0.0621 & 6031 & 0.0624 & 5890 \\
3 & 25.30 & 10.035 & 0.0686 & 6745 & 0.0687 & 6584 & 0.0689 & 6413 & 0.0690 & 6266 & 0.0693 & 6096 \\
4 & 21.27 & 10.035 & 0.0745 & 6864 & 0.0746 & 6699 & 0.0748 & 6584 & 0.0749 & 6455 & 0.0752 & 6226 \\
5 & 16.51 & 10.035 & 0.0875 & 7321 & 0.0876 & 7121 & 0.0880 & 6888 & 0.0881 & 6825 & 0.0885 & 6493 \\
6 & 16.05 & 7.325 & 0.1344 & 8829 & 0.1346 & 8468 & 0.1352 & 8214 & 0.1354 & 8052 & 0.1362 & 7593 \\
7 & 10.44 & 5.575 & 0.2469 & 12476 & 0.2473 & 12112 & 0.2490 & 11493 & 0.2494 & 10997 & 0.2514 & 10272 \\
8 & 9.069 & 4.190 & 0.3892 & 16668 & 0.3899 & 15899 & 0.3933 & 14915 & 0.3941 & 14341 & 0.3981 & 13310 \\
9 & 6.400 & 4.001 & 0.5101 & 20390 & 0.5110 & 19258 & 0.5161 & 18083 & 0.5174 & 17307 & 0.5233 & 16055 \\
10 & 4.196 & 5.550 & 0.5964 & 22829 & 0.5978 & 21409 & 0.6058 & 20349 & 0.6078 & 19292 & 0.6172 & 17772 \\
11 & 7.520 & 3.073 & 0.6519 & 24309 & 0.6532 & 22841 & 0.6606 & 21669 & 0.6624 & 21226 & 0.6710 & 19073 \\
12 & 7.188 & 2.858 & 0.7356 & 26771 & 0.7371 & 25063 & 0.7460 & 23662 & 0.7482 & 22555 & 0.7586 & 20808 \\
\hline
\end{tabular}

Table 2. Ice cylinder $\alpha$ results.

\begin{tabular}{c|cc|cc|cc|cc|cc}
\hline & \multicolumn{2}{|c|}{$-5^{\circ} \mathrm{C}$} & \multicolumn{2}{c|}{$-25^{\circ} \mathrm{C}$} & \multicolumn{2}{c|}{$-45^{\circ} \mathrm{C}$} & \multicolumn{2}{c}{$-65^{\circ} \mathrm{C}$} & \multicolumn{2}{c}{$-85^{\circ} \mathrm{C}$} \\
Cyl. & $\alpha\left(\mathrm{s}^{-1}\right)$ & $\mathrm{C} / \mathrm{E}$ & $\alpha\left(\mathrm{s}^{-1}\right)$ & $\mathrm{C} / \mathrm{E}$ & $\alpha\left(\mathrm{s}^{-1}\right)$ & $\mathrm{C} / \mathrm{E}$ & $\alpha\left(\mathrm{s}^{-1}\right)$ & $\mathrm{C} / \mathrm{E}$ & $\alpha\left(\mathrm{s}^{-1}\right)$ & $\mathrm{C} / \mathrm{E}$ \\
\hline 1 & $5720 \pm 38$ & $0.972 \pm 0.014$ & $5731 \pm 40$ & $1.001 \pm 0.010$ & $5662 \pm 38$ & $1.006 \pm 0.011$ & $5591 \pm 36$ & $1.002 \pm 0.012$ & $5514 \pm 37$ & $1.013 \pm 0.009$ \\
2 & $6539 \pm 46$ & $1.009 \pm 0.012$ & $6439 \pm 45$ & $1.008 \pm 0.009$ & $6331 \pm 42$ & $1.020 \pm 0.009$ & $6181 \pm 41$ & $1.025 \pm 0.009$ & $6091 \pm 42$ & $1.034 \pm 0.013$ \\
3 & $6651 \pm 49$ & $0.986 \pm 0.012$ & $6583 \pm 44$ & $1.000 \pm 0.010$ & $6419 \pm 44$ & $1.001 \pm 0.012$ & $6351 \pm 45$ & $1.014 \pm 0.008$ & $6123 \pm 43$ & $1.004 \pm 0.011$ \\
4 & $6879 \pm 50$ & $1.002 \pm 0.012$ & $6826 \pm 50$ & $1.019 \pm 0.010$ & $6609 \pm 46$ & $1.004 \pm 0.013$ & $6517 \pm 46$ & $1.010 \pm 0.009$ & $6435 \pm 46$ & $1.034 \pm 0.013$ \\
5 & $7373 \pm 51$ & $1.007 \pm 0.010$ & $7268 \pm 49$ & $1.021 \pm 0.010$ & $7061 \pm 47$ & $1.025 \pm 0.010$ & $6916 \pm 47$ & $1.013 \pm 0.013$ & $6587 \pm 46$ & $1.014 \pm 0.013$ \\
6 & $8875 \pm 64$ & $1.005 \pm 0.009$ & $8650 \pm 67$ & $1.021 \pm 0.011$ & $8367 \pm 64$ & $1.019 \pm 0.012$ & $8087 \pm 57$ & $1.004 \pm 0.012$ & $7747 \pm 54$ & $1.020 \pm 0.012$ \\
7 & $12503 \pm 106$ & $1.002 \pm 0.009$ & $12102 \pm 96$ & $0.999 \pm 0.010$ & $11470 \pm 85$ & $0.998 \pm 0.011$ & $10903 \pm 81$ & $0.991 \pm 0.010$ & $10105 \pm 80$ & $0.984 \pm 0.011$ \\
8 & $16841 \pm 139$ & $1.010 \pm 0.009$ & $15985 \pm 113$ & $1.005 \pm 0.010$ & $15320 \pm 110$ & $1.027 \pm 0.009$ & $14180 \pm 100$ & $0.989 \pm 0.008$ & $13391 \pm 100$ & $1.006 \pm 0.011$ \\
9 & $20025 \pm 159$ & $0.982 \pm 0.010$ & $19326 \pm 148$ & $1.004 \pm 0.010$ & $18344 \pm 142$ & $1.014 \pm 0.011$ & $16943 \pm 133$ & $0.979 \pm 0.011$ & $15855 \pm 113$ & $0.988 \pm 0.011$ \\
10 & $22828 \pm 200$ & $1.000 \pm 0.014$ & $21398 \pm 190$ & $0.999 \pm 0.012$ & $20056 \pm 180$ & $0.986 \pm 0.015$ & $19087 \pm 160$ & $0.989 \pm 0.017$ & $17762 \pm 157$ & $0.999 \pm 0.014$ \\
11 & $24412 \pm 297$ & $1.004 \pm 0.020$ & $23074 \pm 262$ & $1.010 \pm 0.015$ & $21363 \pm 260$ & $0.986 \pm 0.017$ & $20266 \pm 211$ & $0.955 \pm 0.013$ & $19077 \pm 207$ & $1.000 \pm 0.021$ \\
12 & $26706 \pm 389$ & $0.998 \pm 0.020$ & $25960 \pm 362$ & $1.036 \pm 0.018$ & $23446 \pm 269$ & $0.991 \pm 0.015$ & $22144 \pm 280$ & $0.982 \pm 0.015$ & $20901 \pm 318$ & $1.004 \pm 0.019$ \\
\hline
\end{tabular}

\subsection{Idealised PWR Flooded Fuel Flask}

A simplified model of PWR fuel elements in a steel cask with all internal spaces filled with water or ice was used to simulate the effect of a fuel flask cooling; the water changes to ice (with respect to density and thermal scattering data) from the outside inward. Decreasing the density outside the fuel element significantly increases k-effective due to increased element-to-element interaction. Decreasing the density within the fuel element significantly decreases k-effective due to decreased neutron moderation. Using ice nuclear data outside the fuel element slightly increases k-effective due to decreased moderation thereby increasing element-to-element interaction. Using ice nuclear data, within the fuel element, decreases k-effective due to decreased moderation. Therefore, the most critical configuration occurs where there is water within the fuel elements and ice outside the fuel elements.

\section{Conclusions}

A continuous energy (BINGO) nuclear data library has been produced for the MONK Monte Carlo code enabling direct criticality calculations for temperatures down to 193 K. Comparison of MONK results against low temperature calculation benchmarks and experimental data shows good agreement. Further calculations for application cases show the impact of modelling liquid and solid $\mathrm{H}_{2} \mathrm{O}$ with appropriate water and ice thermal scattering data as well as material density.

\section{Acknowledgements}

The authors acknowledge the support for this work from EDF Energy, the UK Office for Nuclear Regulation and the UK Ministry of Defence.

\section{References}

[1] S. D. Richards et al., ANE 82, 63-73 (2015)

[2] T. Ware et al., ND2019, EPJ WoC, Paper I224, t.b.p.

[3] A. C. Kahler (ed.), LA-UR-17-20093, (2016)

[4] M. Mattes and J. Keinert, INDC(NDS)-0470 (2005)

[5] M. Mattes and J. Keinert, INDC(NDS)-0475 (2005)

[6] D. A. Brown et al., NDS 148, 1-142 (2018)

[7] D. I. Page, B. C. Haywood, AERE-R 5778 (1968)

[8] L. Torres et al., NIM B 251304305 (2006)

[9] EXFOR database; www-nds.iaea.org/exfor/.

[10] D. Heinrichs, ND2016, EPJ WoC 146, 13004 (2017)

[11] E. G. Silver, NSE 34, 275-284 (1968)

[12] J. C. Wagner and G. Radulescu, NEA Expert Group on Burn-up Credit (2008)

[13] O. Cabellos, JEF/DOC-1844, JEFF Meetings, Paris, France (2017)

[14] E. G. Silver, Oak Ridge National Laboratory, ORNLTM-1204 (1965) 